\title{
Update Epilepsie
}

B. J. Steinhoff

Epilepsiezentrum Kork, Kehl-Kork

\section{Schlüsselwörter}

Epilepsie, Klassifikation, Diagnostik, Therapie, Update

\section{Zusammenfassung}

In der Epileptologie hat sich eine intensive Diskussion zur Klassifikation epileptischer Anfälle und Syndrome entwickelt, die noch nicht abgeschlossen ist. Sie reflektiert die erheblichen Fortschritte, die im Verständnis der Ätiologie und Pathophysiologie gewonnen wurden, aber auch den lange noch nicht abgeschlossenen Erkenntniszugewinn. Weitere Modifikationen sind daher zwingend zu erwarten. Die diagnostischen Möglichkeiten haben sich weiter verbessert. Hauptproblem ist, dass sie nicht angemessen angewendet werden. Die Grundsätze der Epilepsietherapie haben sich in letzter Zeit nicht verändert. Es hat sich gezeigt, dass Lamotrigin und Levetiracetam zu den Standardantikonvulsiva werden. Neueste Antikonvulsiva haben zum Teil überzeugt. Leider wird ihre nachhaltige Anwendung in Deutschland im Gegensatz zum Rest der Welt durch die Auswirkungen des Arzneimittelmarktneuordnungsgesetzes praktisch verhindert. Neurostimulationsverfahren bieten interessante Perspektiven, aber nicht mehr.

\section{Keywords}

Epilepsy, classification, diagnostics, therapy, update

\section{Summary}

There has been an ongoing and vivid discussion about the classification of epileptic seizures and syndromes that has not been finished yet. It reflects the substantial progress of understanding etiology and pathophysiology but also the preliminary character of this knowledge. Further modifications are to be expected. The diagnostic possibilities have advanced. The major problem is that these ameliorations are not widely used. The basic rules of anticonvulsant treatment remained the same. It is apparent that lamotrigine and levetiracetam are the modern standard anticonvulsant drugs. The latest anticonvulsant drugs are only partial improvements. Unfortunately, their sustained availability is prevented by the consequences of new regulatory laws in Germany. Neurostimulation methods offer interesting perspectives, not more and not less.

Update epilepsy

Nervenheilkunde 2016; 35: 658-663

eingegangen am: 10. Mai 2016

angenommen am: 2. Juni 2016
Diagnostik und Therapie der Epilepsien sind einem stetigen Wandel unterzogen. Dabei ist das Phänomen epileptischer Anfälle seit Jahrtausenden so unverändert wie häufig. Die Wahrnehmung und Beschreibung richtet sich nach der genaueren Dokumentation mittels Video-EEG und dem besseren pathophysiologischen Verständnis. Ähnlich ist es bei epilepti- schen Syndromen. Gängige und häufige Syndrome wie die Epilepsie mit zentrotemporalen epilepsietypischen Mustern oder das Dravet-Syndrome waren vor 30 Jahren nicht bekannt. Elektroklinische Zusammenhänge wie die hochfrequenten epilepsietypischen Muster bei kortikalen Dysplasien konnte man nicht erkennen, weil die bildgebende Diagnostik die De- tektion kortikaler Dysplasien noch gar nicht erlaubte.

So ändert sich vor allem unser Verständnis vom Symptom Anfall und von der Krankheit Epilepsie. Je besser wir die $\mathrm{Zu}-$ sammenhänge verstehen, desto besser sollten unsere therapeutischen Möglichkeiten sein.

Diese kurze Zusammenfassung der klinisch wesentlichen und auf die Erwachsenenepileptologie bezogenen Neuerungen der letzten Zeit widmet sich daher den neuen Klassifikationsansätzen, den aktuellen diagnostischen Möglichkeiten mit den entsprechenden Qualitätsstandards und gibt einen Überblick über wichtige aktuelle Publikationen zur Epilepsietherapie.

\section{Klassifikation epileptischer Anfälle und Syndrome}

Die bislang gültige offizielle Klassifikation epileptischer Anfälle datiert aus dem Jahre 1981 (1), die Klassifikation epileptischer Syndrome wurde 1989 publiziert (2). Diese bis heute in der Welt der Neurologie angekommenen Klassifikationen basieren naturgemäß auf Konzepten, die überwiegend aus der Zeit vor der Einführung moderner Bildgebungsmethoden oder molekularbiologischer Konzepte stammen. Selbstverständlich haben die damals verantwortlichen Autoren vorausgesehen, dass die Entwicklung neuer Untersuchungsmethoden Änderungen der Klassifikationen erforderlich machen würden. Es sind tatsächlich in den Folgejahren immer wieder einmal Verbesserungsvorschläge publiziert worden, die zum Teil heftig kritisiert wurden und kaum Verbesserungen mit sich brachten, die in irgendeiner Weise von praktischem Wert gewesen wären (3-5).

Ein schon vor einiger Zeit publiziertes Glossar zu epileptischen Anfällen (6) hat immerhin hinsichtlich der Anfallsklassifikationen auch konzeptionell Verbesserungen erbracht, die zum Teil in die aktuell 
publizierte und verbindliche revidierte Terminologie zur Einteilung von epileptischen Anfällen und Epilepsien (7) eingegangen sind. Die bisherigen Klassifikationen gingen hinsichtlich epileptischer Syndrome von der grundsätzlichen pathophysiologischen Dichotomie zwischen fokaler und generalisierter Epileptogenese aus. Dazu kam hinsichtlich der epileptischen Syndrome eine ätiologische Unterteilung in idiopathisch, symptomatisch und kryptogen (wahrscheinlich symptomatisch). Diese Einteilung wird mit der neuen Klassifikation aufgegeben. Sie verzichtet auf die Begriffe "fokal“ und „generalisiert", da schon in der jüngeren Vergangenheit, im Wesentlichen befruchtet durch die neurophysiologischen, bildgebenden und molekulargenetischen neuen Erkenntnisse, das Konzept eines Kontinuums der Epileptogenese favorisiert wurde (8), das individualspezifisch verschiedene, möglicherweise im Einzelfall nahezu gleichrangige, für den Phänotyp epileptischer Anfälle notwendige ätiologische Voraussetzungen diskutierte. Die Klassifikation epileptischer Anfälle behält die Begriffe "fokal“ und "generalisiert" bei, wobei generalisierte Anfälle als Resultat einer bilateralen Netzwerkstörung aufgefasst werden, während fokale Anfälle einem unihemisphärischen Netzwerk entspringen.

Die Klassifikation generalisierter Anfälle wurde vereinfacht. Hinsichtlich der fokalen Anfälle ist von entscheidender Bedeutung, dass die schon immer heftig kritisierte Unterteilung in einfach bzw. komplexfokale Anfälle aufgegeben wird und stattdessen entsprechend des Glossars epileptischer Anfälle (6) eine deskriptive Beschreibung eingefordert wird. Fokale Anfälle unterteilen sich demnach in fokale Anfälle mit motorischen Symptomen, mit sensibel/ sensorischen Symptomen, mit vegetativen Symptomen, mit psychischen Symptomen oder mit dyskognitiven Symptomen (am ehesten dem klassischen komplex-fokalen oder psychomotorischen Anfall entsprechend) sowie in sekundär-generalisierte Anfälle ( $\triangleright$ Kasten).

Die Klassifikation der Epilepsien gliedert sich in elektroklinische Syndrome und in Konstellationen, womit Krankheiten auf dem Boden spezifischer Läsionen oder anderer Ursachen gemeint sind. Von großer

\section{Klassifikation epileptischer Anfälle}

\section{Generalisierte Anfälle \\ tonisch-klonisch (in jeder Kombination) \\ Absence \\ - typisch \\ - atypisch \\ - mit speziellen Merkmalen \\ - myoklonisch Absence \\ - Lidmyoklonien mit Absence \\ myoklonisch \\ - myoklonisch \\ - myoklonisch-atonisch \\ - myoklonisch-tonisch}

klonisch

tonisch

atonisch

\section{Fokale Anfälle}

mit motorischen Symptomen mit sensibel/sensorischen Symptomen mit vegetativen Symptomen mit psychischen Symptomen mit dyskognitiven Symptomen mit Generalisierung

\section{Unbekannt}

epileptische Spasmen

Bedeutung ist in der neuen Klassifikation der natürliche Verlauf der Erkrankung. Dies wird unterstrichen durch die Wiedereinführung des Begriffs der epileptischen Enzephalopathie. Prognostisch allzu apodiktisch wegweisende Vokabeln wie „benigne" oder „katastrophal“ werden dagegen weitgehend (bis auf das Syndrom der Rolando-Epilepsie) gestrichen. Eine weitere Systematik hinsichtlich der Epilepsien entsteht dadurch, dass die elektroklinischen Syndrome altersabhängig geordnet werden. Der Kasten gibt die neue Klassifikation elektroklinischer Syndrome und anderer Epilepsien wieder.

\section{Moderne leitliniengerechte Diagnostik}

Bei einer Erkrankung, die durch gelegentliche paroxysmale Symptome gekennzeich- net ist, spielt die Anamnese womöglich noch eine größere Rolle als bei anderen Konstellationen in der Medizin. Bei der Diagnose von Epilepsien ist es eminent wichtig, die zur Verfügung stehenden Verfahren konsequent und auf adäquatem $\mathrm{Ni}$ veau einzusetzen. Dabei genügt es keineswegs, sich auf die Ergebnisse von Routineuntersuchungen $\mathrm{zu}$ verlassen. Es ist bekannt, dass nach mehreren normalen Routine-EEG-Ableitungen keine weitere diagnostisch relevante Aussage mehr zu erwarten ist, während bei entsprechender diagnostischer Fragestellung die frühzeitige Dokumentation des Befundes unter Ausnutzung der hohen zeitlichen Auflösung des EEG, also mit Langzeit-Video-EEG unbedingt anzustreben ist. Bedeutsam ist leider, dass in den letzten Jahren das Niveau der EEG-Befundung vielerorts nachgelassen hat. In spezialisierten Zentren ist festzustellen, dass vor allem falsch positive Befunde zunehmen. Zahlreich sind die Patientenkarrieren, bei denen eine unzureichende Diagnostik und ein vorschnelles Festlegen auf eine so schwerwiegende Diagnose wie die einer Epilepsie zu jahrzehntelangen Fehlbehandlungen geführt haben. Bei dem Verdacht auf eine fokale Epilepsie ist dem MRT zur Ursachensuche größte Bedeutung zuzuerkennen. Die bildgebende Diagnostik hat differenzialätiologisch einen ungeheuren Fortschritt mit sich gebracht und muss im Bedarfsfall unbedingt zum Einsatz kommen. Dabei hat sich leider gezeigt, dass eine individualspezifische und an die syndromatologische Hypothese angepasste MRT-Diagnostik häufig unterbleibt und darüber hinaus Radiologen oft nicht in der Lage sind, epilepsierelevante Befunde wie mesiale temporale Sklerosen oder kortikale Dysplasien zu erkennen; Befunde, wie sie in der Ära vor dem MRT noch gar nicht intra vitam zu erheben gewesen waren. Deshalb ist es unbedingt notwendig, dass sich der Kliniker selbst mit den Originalbefunden beschäftigt. Leider werden auch auf diesem Gebiet zum Teil erschreckende Entwicklungen offenbar: Zum einen werden klare Befunde oft übersehen, weil entweder nicht leilinienkonforme Schichtungen und Orientierungen (9) durchgeführt oder aber dennoch nur durch Experten erkannt werden (10), zum anderen werden selbst bei intellektuell in- 
takten Erwachsenen, die wegen schwer behandelbarer Epilepsien in Epilepsiezentren überwiesen werden, noch nicht einmal MRT-Untersuchungen durchgeführt (11). In den letzten Jahren hat die Genetik der Epilepsien dramatische Fortschritte erzielt. Die Veranlassung einer kranialen MRT ist nur dann nicht obligat, wenn durch den klinischen und elektroenzephalographischen Befund nicht der geringste Zweifel an einer idiopathischen generalisierten Epilepsie besteht und deren medikamentöse Therapie offensichtlich erfolgreich ist. Bei Verdacht auf eine infektiöse symptomatische Genese ist eine Liquoranalyse erforderlich, die grundsätzlich zu empfehlen ist. Die Tabelle gibt einen Überblick über die notwendige Diagnostik bei epileptischen Anfällen und Epilepsien.

\section{Aktueller Therapiestandard}

Wird eine medikamentöse Epilepsietherapie initiiert, sollte man sich der großen Verantwortung bewusst sein, die mit dieser Entscheidung übernommen wird. Ein Großteil der Patienten wird eine lebenslange Therapie vor sich haben (12). Wesentliche Wirksamkeitsunterschiede zwischen den verfügbaren Antikonvulsiva bestehen nicht (12). Welches Antikonvulsivum auch immer gewählt wird, die Wahrscheinlichkeit einer zufriedenstellenden Wirksamkeit beträgt etwa $70 \%$, und zwar mit alten wie mit neuen Antikonvulsiva $(13,14)$. Wenn also ein Patient erst einmal anfallsfrei ist, aber Störwirkungen im Verlauf auftreten, ist es oft kaum möglich, diese Patienten von einer medikamentösen Umstellung zu überzeugen. Da wir heute die Möglichkeit haben, zur Vorbeugung von Langzeittoxizität, von Interaktionsproblemen bei Kombination mit anderen Antikonvulsiva, aber auch von anderen Medikamenten bei Auftreten behandlungsbedürftiger anderer Erkrankungen akut und im Langzeitverlauf und zur Verbesserung der Lebensqualität durch eine geringere Toxizität entsprechende Antikonvulsiva auszuwählen, sollten wir dies auch unbedingt tun. Vor allem deshalb werden in den Leitlinien Lamotrigin (LTG) und Levetiracetam (LEV) als Antikonvulsiva der ersten Wahl vor den ebenfalls zur Monotherapie zugelassenen Carbamazepin (CBZ), Gabapentin (GBP), Phenytoin (PHT), Oxcarbazepin (OXC), Topiramat (TPM), Valproinsäure (VPA) und Zonisamid (ZNS) eingeordnet (15).

\section{Elektroklinische Syndrome und andere Epilepsien}

\section{Elektroklinische Syndrome, nach Manifestationsalter geordnet \\ Neugeborenenzeit \\ - benigne familiäre neonatale Epilepsie \\ - frühe myoklonische Enzephalopathie \\ - Ohtahara-Syndrom}

Kleinkindalter

- Epilepsie der frühen Kindheit mit migratorischen fokalen Anfällen

- West-Syndrom

myoklonische Epilepsie der frühen Kindheit

benigne frühkindliche Epilepsie

benigne familiäre frühkindliche Epilepsie

Dravet-Syndrom

myoklonische Enzephalopathie bei nicht progredienten Störungen

\section{Kindheit}

- fiebergebundene Anfälle plus (können in der frühen Kindheit bzw. im Kleinkinde-

salter beginnen)

- Panayiotopoulos-Syndrom

- Epilepsie mit myoklonisch-atonischen Anfällen

- benigne Epilepsie mit zentrotemporalen Spikes (Rolando-Epilepsie)

- autosomal-dominante Frontallappenepilepsie

- spät beginnende kindliche Okzipitallappenepilepsie
- Epilepsie mit myoklonischen Absencen

- Lennox-Gastaut-Syndrom

- epileptische Enzephalopathie mit kontinuierlichen Spiek-und-Wave-Entladungen im Schlaf (CSWS)

- Landau-Kleffner-Syndrom

rindliche Absencen-Epilepsie

Adoleszenz - Erwachsenenalter

- juvenile Absencen-Epilepsie

- juvenile myoklonische Epilepsie

- Epilepsie mit nur generalisierten tonischklonischen Anfällen

- progressive Myoklonusepilepsien

- autosomal-dominante fokale Epilepsie mit akustischen Merkmalen

- andere familiäre Temporallappenepilepsien

\section{Weniger spezifische Alters-} beziehung

- familiäre fokale Epilepsie mit variablen Herden (Kindheit bis Erwachsenenalter)

- Reflexepilepsien

Unverwechselbare Konstellationen

- mesiale Temporallappenepilepsie mit Hippocampussklerose

- Rasmussen-Syndrom

- gelastische Anfälle bei hypothalamischen Hamartomen
- Hemikonvulsion-Hemiplegie-Epilepsie (-Syndrom)

Epilepsien, die nicht in diese Kategorien passen, können zunächst auf der Basis des Vorhandenseins oder Fehlens einer bekannten strukturellen oder metabolischen Störung (vermutliche Ursache) und dann auf der Basis des primären Anfallsbeginns (generalisiert vs. fokal) unterschieden werden.

\section{Epilepsien aufgrund von und eingeteilt nach strukturell- metabolischen Ursachen}

- Malformationen der kortikalen Entwicklung (z. B. Hemimegalenzephalie, Heterotopien)

- neurokutane Syndrome (z. B. TuberöseSklerose-Komplex, Sturge-Weber-Syndrom)

- Tumoren

- Infektionen

- Traumen

- Angiome

- perinatale Infarkte

- andere Infarkte

\section{Epilepsien unbekannter Ursache}

Zustände mit epileptischen Anfällen, die traditionell nicht als eine Epilepsieform per se betrachtet werden

- benigne neonatale Anfälle

- fiebergebundene Anfälle

( „Fieberkrämpfe“) 
Wegen der besseren Wirksamkeit im Vergleich zu LTG und der besseren Verträglichkeit im Vergleich zu TPM wird bei generalisierten Epilepsien immer noch VPA als Mittel der ersten Wahl eingestuft. LEV wird in der Praxis sehr oft verwendet (16), ist aber offiziell zur Monotherapie nicht zugelassen. Die Mitglieder der Deutschen Gesellschaft für Epileptologie erklärten in einer internetbasierten Umfrage in Übereinstimmung mit der klinischen Erfahrung und dem medizinischen Fachwissen zur Pharmakologie von LEV und entgegen der Zulassungssituation, dass sie mehrheitlich (75\%) LEV auch in Monotherapie bei generalisierten Epilepsien einsetzen, es auch bei anderen Syndromen über die juvenile myoklonische Epilepsie hinaus nutzen (81\%) und es ablehnen (84\%), zum Schein der Wahrung des Zulassungsstatus eine Kombination von LEV mit einem „Pseudoplacebo" zu geben, also einem vermutlich kaum wirksamen, niedrig dosierten anderen Antikonvulsivum zusammen (15).

Bei Mädchen und Frauen muss wegen der unzweifelhaft erhöhten Teratogenität mittlerweile der Nachweis geführt werden, dass Alternativen versucht worden sind. Ist VPA das einzig wirksame Medikament, empfiehlt sich die Dokumentation einer ausführlichen Aufklärung über die Teratogenität und die Möglichkeit einer kognitiven Minderleistung in den ersten 6 Lebensjahren bei Kindern, deren Mütter in der Schwangerschaft hochdosiert VPA eingenommen haben $(17,18)$.

Grundsätzlich wird initial eine Monotherapie angestrebt. Sie ist einfach beurteilbar, in den meisten Fällen ausreichend wirksam und im Hinblick auf potenzielle Interaktionen weniger problematisch (15, 19). In letzter Zeit mehren sich Hinweise darauf, dass durch sinnvolle Kombinationen noch wesentliche Verbesserungen dann erzielt werden können, wenn ausdosierte Monotherapien nicht zur stets prinzipiell anzustrebenden nachhaltigen Anfallsfreiheit geführt haben (20-22). Darüber hinaus ist davon auszugehen, dass die Therapietreue gerade von Patienten mit Erstdiagnose und Erstbehandlung bei einer initialen Monotherapie besser ist.

Tab. Diagnostik bei epileptischen Anfällen (nach 10)

\begin{tabular}{|c|c|}
\hline Diagnostische Maßnahme & Implikation / Befunde \\
\hline $\begin{array}{l}\text { Eigen-/Fremdanamnese, } \\
\text { gegebenenfalls Videoaufzeichnung }\end{array}$ & $\begin{array}{l}\text { Sicherung der Diagnose } \\
\text { - Analyse der Anfallsphänomenologie } \\
\text { - Differenzierung gegenüber kardiovaskulären Synkopen, } \\
\text { Hypoglykämie, Narkolepsie, Migräne, transitorisch ischä- } \\
\text { mischen Attacken, dissoziativen (psychogenen) Anfällen }\end{array}$ \\
\hline $\begin{array}{l}\text { Schlafentzugs-/ Langzeit-EEG, } \\
\text { Spezialelektroden } \\
\text { Video-EEG }\end{array}$ & $\begin{array}{l}\text { Ergänzende Aussagekraft } \\
\text { - Nachweis epilepsietypischer Potenziale } \\
\text { - bilateral synchrone Spike-Wave- oder Polyspike-Wave- } \\
\text { Komplexe bei idiopathischen generalisierten Epilepsien } \\
\text { (häufig) } \\
\text { - Spikes oder Sharp-Waves bei Epilepsien fokalen Ur- } \\
\text { sprungs (seltener) } \\
\text { - Erhöhte Nachweisrate epilepsietypischer Potenziale } \\
\text { - Anfallsaufzeichnung zur Korrelation des klinischen und } \\
\text { des EEG-Befundes } \\
\text { - Nutzung der besseren zeitlichen Auflösung mit Erfassung } \\
\text { von Müdigkeit und Schlaf }\end{array}$ \\
\hline MRT & $\begin{array}{l}\text { Nachweis / Ausschluss symptomatischer Ursachen:Neoplas- } \\
\text { men, Traumata, Infektionen, vaskuläre Läsionen, Missbil- } \\
\text { dungen, mesiale temporale Sklerose, fokale kortikale Dys- } \\
\text { plasien }\end{array}$ \\
\hline Liquoranalyse & $\begin{array}{l}\text { Nachweis / Ausschluss symptomatischer Ursachen: Infektio- } \\
\text { nen, Autoimmunerkrankungen (z. B. limbische Enzephalitis, } \\
\text { Vaskulitiden) }\end{array}$ \\
\hline Blutanalyse & $\begin{array}{l}\text { Ergänzende Aussagekraft } \\
\text { - CK-Erhöhung nach bilateral konvulsivem Anfall } \\
\text { - Prolaktinerhöhung bis max. } 1 \text { h nach Anfällen, nur sinn- } \\
\text { voll bei intraindividuellem Vergleich mit tageszeitlich ent- } \\
\text { sprechendem Vergleichswert im anfallsfreien Intervall }\end{array}$ \\
\hline $\begin{array}{l}\text { Kardiologische Zusatzdiagnostik ein- } \\
\text { schließlich Kipptischuntersuchungen }\end{array}$ & $\begin{array}{l}\text { Positive Synkopendiagnostik und Synkopenklassifikation, } \\
\text { wesentlich zur Differenzialdiagnose ungeklärter paroxysma- } \\
\text { ler Bewusstseinsstörungen }\end{array}$ \\
\hline
\end{tabular}

\section{Neues zu LTG und LEV als Standardantikonvulsiva der dritten Generation}

LTG und LEV gelten schon seit einiger Zeit als Standardantikonvulsiva zur Erstbehandlung Erwachsener mit fokaler Epileptogenese (15). Praxisstudien sind allerdings rar. Vor kurzem wurden zwei wichtige Arbeiten publiziert, die LEV einerseits mit VPA und CBZ und andererseits mit LTG verglichen $(23,24)$. Die Vergleichsstudie zu VPA und CBZ entsprach einer unverblindeten randomisierten Parallelgruppenstudie über eine Dauer von 52 Wochen. Schon früher ist das Design eines Vergleichs mit einer Standardtherapie bei Patienten mit fokalen bzw. generalisierten Anfällen und damit im Ver- gleich mit entweder CBZ oder VPA als etablierter Goldstandard eingesetzt worden $(25,26)$. Solche Studien zielen darauf ab, dass mit hoher Wahrscheinlichkeit der überwiegende Teil der neu diagnostizierten und in die Studie aufgenommenen Patienten eher mit VPA behandelt werden, wenn es sich um generalisierte Anfälle handelt und eher mit CBZ im Falle von fokalen epileptischen Anfällen. Allerdings ist die Trennschärfe nicht absolut. Die primäre Zielvariable war die Quote an Behandlungsabbrechern. Sekundäre Zielvariablen umfassten die Zeit bis zum ersten Anfall, die Rate der Behandlungsabbrüche nach 6 und 12 Monaten und die Rate anfallsfreier Patienten nach 6 und 12 Monaten. Insgesamt wurden 1698 Patienten randomisiert. Die Zeit bis zum Abbruch der Behandlung 
war zwischen LEV und den Standardantikonvulsiva CBZ und VPA statistisch nicht signifikant unterschiedlich. Bei der Zeit bis zum ersten Anfall ergab sich ein statistisch signifikanter Vorteil der Standardantikonvulsiva, wenn diese zusammen betrachtet wurden, nicht aber von VPA oder CBZ alleine. Nach 6 Monaten waren unter LEV $59,8 \%$ und unter Standardantikonvulsiva 64,5\% der Patienten anfallsfrei. Nach 12 Monaten betrugen die Anfallsfreiheitsquoten für LEV 53,9\% und für Standardantikonvulsiva 59,9\%. Die Unterschiede waren statistisch nicht signifikant.

Positiv formuliert hat sich zeigen lassen, dass LEV mit den seit Jahrzehnten etablierten Standardantikonvulsiva mithalten kann und als „Breitband-Antikonvulsivum“ sowohl bei fokalen als auch bei generalisierten Epilepsien eingesetzt werden kann. Es ist bedauerlich, dass die Monotherapie bei generalisierten Epilepsien einer Off-label-Therapie entspricht, was die Mehrheit der Epilepsieexperten in Deutschland in der Praxis allerdings ignoriert (16). VPA und CBZ sind ausgesprochen wirksam und im Initialverlauf von 52 Wochen auch durchaus gut verträgliche Medikamente ebenso wie LEV.

Die Vergleichsstudie gegen LTG umfasste einen Zeitraum von 26 Wochen. Es handelte sich um eine unverblindete randomisierte Parallelgruppenstudie. Eingeschlossen wurden Patienten ab einem Alter von mindestens 12 Jahren, bei denen erste fokale oder generalisierte Anfälle aufgetreten waren. Die angestrebten Erhaltungsdosen betrugen für LEV $2000 \mathrm{mg}$ und für LTG $200 \mathrm{mg}$ pro Tag. Die primäre Zielvariable war der Prozentsatz anfallsfreier Patienten in den ersten 6 Wochen nach Randomisierung. Sekundäre Zielvariablen waren die Anfallsfreiheit während der letzten 16 Wochen der Studie, Anfallsfreiheit während der kompletten Studienperiode, die Retentionszeit, die Verträglichkeit und die Lebensqualität. Insgesamt gingen 409 Patienten in die Studie ein (206 unter LEV und 203 unter LTG). Es wurden keine signifikanten Unterschiede bezüglich der Anfallsfreiheitsraten, der Retentionsrate und der Lebensqualität gefunden, wobei sich die Retentionsrate 63,1\% unter LEV im Vergleich zu 71,4\% unter LTG doch deutlich unterschied und gerade eben nicht signifikant zugunsten von LTG ausfiel $(\mathrm{p}=0,07)$. Hinsichtlich Störwirkungen waren zwischen beiden Substanzen kaum signifikante Unterschiede festzustellen. Lediglich Müdigkeit und Aggression wurden jeweils statistisch signifikant häufiger unter LEV beobachtet.

Bei älteren Patienten, die anfälliger für Störwirkungen sind, lassen sich die Vorteile von LTG und LEV deutlicher zeigen: Eine Vergleichsstudie zwischen Monotherapien mit LTG, GBP und unretardiertem CBZ an 593 Patienten im höheren Lebensalter mit neu aufgetretenen Epilepsien (27) und Tageszieldosen von $150 \mathrm{mg}$ LTG, $1500 \mathrm{mg}$ GBP und $600 \mathrm{mg}$ CBZ ergab, dass unter CBZ wiederum aufgrund schlechterer Verträglichkeit signifikant mehr Therapien abgebrochen wurden. Tendenziell, aber nicht signifikant schnitt LTG besser ab als GBP. Wichtig ist natürlich, dass bei Einsatz eines Antikonvulsivums auch die bestmögliche Formulierung benutzt wird, auch und gerade bei CBZ (28). Eine skandinavische Studie verglich bei älteren Patienten LTG mit diesmal retardiertem CBZ und konnte keine statistisch signifikanten Unterschiede nachweisen (29). Eine sehr wichtige Arbeit zum Thema wurde vor kurzem publiziert (30). Hierbei handelte es sich um eine randomisierte, doppelblinde Parallelgruppenstudie, die an 47 Krankenhäusern in Deutschland, Österreich und der Schweiz zwischen Januar 2007 und August 2011 bei Patienten über 60 Jahren und neu aufgetretener, behandlungsbedürftiger Epilepsie durchgeführt wurde. Die Patienten wurden mit LTG, LEV oder retardiertem CBZ behandelt. Statistisch verglichen wurde LEV mit den anderen beiden Substanzen. Die Medikation wurde innerhalb von 6 Wochen eindosiert. Die Zieldosen betrugen für CBZ $400 \mathrm{mg}$, für LTG $100 \mathrm{mg}$ und für LEV 1000 mg. Die primäre Zielvariable war die Retention nach 58 Wochen. Rekrutiert wurden jeweils um 120 Patienten. Die Retentionsrate unter LEV war signifikant höher als unter CBZ und nicht statistisch unterschiedlich zu LTG. Ausschlaggebend war die schlechtere Verträglichkeit von CBZ.

\section{Neues zu neuesten Antikonvulsiva}

Die seit 2008 eingeführten Antikonvulsiva finden hinsichtlich ihrer Erfolgsaussichten veränderte Bedingungen vor: Nach 1992 eingeführte Antikonvulsiva sind generisch verfügbar, also deutlich preiswerter. Ein neu eingeführtes und teures Medikament muss daher besonders attraktive Therapiealternativen bieten, um sich durchsetzen zu können. Dazu kommt, dass das Arzneimittelmarktneuordungsgesetz (AMNOG), das nach Einführung von Lacosamid (LCM) zur Anwendung kam, dazu geführt hat, dass sowohl Retigabin (RTG) als auch Perampanel (PER) aufgrund eines laut Gemeinsamem Bundesausschuss nicht vorhandenen $\mathrm{Zu}-$ satznutzens mittlerweile von den Herstellern in Deutschland außer Vertrieb genommen wurden. Das führt zu der absurden Situation, dass in Europa zugelassene Antikonvulsiva für deutsche Patienten nur in seltensten Ausnahmen noch zur Verfügung stehen, deutsche Patienten also potenziell schlechtere Behandlungschancen haben. Die Generation der genannten neuesten Antikonvulsiva lässt sich in 2 Gruppen aufteilen: Eslicarbazepinacetat (ESL) ist ein Analogon von Carbamazepin und Oxcarbazepin, Brivaracetam (BRV) von Levetiracetam. Für beide wird als Unterscheidungsmerkmal aufgeführt, sie böten zusätzliche (ESL) bzw. höherpotent umgesetzte (BRV) Wirkmechanismen $(31,32)$. LCM, RTG und PER hingegen haben von Anfang an neue Wirkmechanismen offeriert $(33,34)$. Die Nutzen-Risiko-Beurteilung fällt unterschiedlich aus. RTG hat erhebliche Verträglichkeitsrisiken und führt unter anderem in seltenen Fällen zu Blauverfärbungen von Retina oder Fingernägeln (34). Die Wirksamkeit war in einer offenen Studie in Deutschland mäßig, die Abbruchrate hoch (35). Die Erfolgsraten als Zusatztherapie entsprechend des Zulassungsbereichs sind für ESL, LCM und PER in mancher offenen Beobachtung durchaus beachtlich (36-39), keines der genannten neuen Antikonvulsiva wird allerdings die Epilepsietherapie revolutionieren. Um die Wirksamkeit und Verträglichkeit von BRV zu beurteilen, ist der Beobachtungszeitraum nach der Markteinführung noch zu kurz.

\section{Neue Neurostimulations- verfahren}

Gerade in Deutschland erfreuen sich aufgrund der geschilderten Probleme, die das 
AMNOG verursacht, neue Neurostimulationsverfahren großer Beachtung. Neben den zugelassenen invasiven Verfahren, der invasiven Vagusnervstimulation und der Tiefenhirnstimulation, wurden nicht invasive Verfahren erprobt und in Studien untersucht, vor allem die externe Vagus- und die externe Trigeminusstimulation $(40,41)$. Beide Verfahren erfordern eine hohe Adhärenz der Patienten, da mehrstündige Anwendungen pro Tag vorgesehen sind. Von Vorteil ist sicher die Reversibilität und praktisch fehlende Gefahr von bleibenden oder gefährdenden Störwirkungen.

\section{Interessenkonflikt}

Bernhard J. Steinhoff hat honorarpflichtig Vorträge für Eisai und UCB gehalten. Er hat Beraterfunktionen für die Firmen Actelion, Bial, B. Braun, Melsungen, Desitin, Eisai und UCB wahrgenommen.

\section{Literatur}

1. Commission on Classification and Terminology of the International League against Epilepsy. Proposal for revised clinical and electroencephalographic classification of epileptic seizures. Epilepsia 1981; 22: 498-501.

2. Commission on Classification and Terminology of the International League against Epilepsy. Proposal for revised classification of epilepsies and epileptic syndromes. Epilepsia 1989; 30: 389-99.

3. Engel J. A proposed diagnostic scheme for people with epileptic seizures and with epilepsy: report of the ILAE Task Force on Classification and Terminology. Epilepsia 2001; 42: 796-803.

4. Engel J. Report of the ILAE Classification Core Group. Epilepsia 2006; 47: 1558-1568.

5. Steinhoff B, Krämer G. Revidierte Terminologie und neue Konzepte zur Einteilung von epileptischen Anfällen und Epilepsien. Neue Empfehlungen. Muss das wirklich sein?. Z Epileptol 2010; 23: 225-226.

6. Blume WT et al. Glossary of descriptive terminology for ictal semiology: report of the ILAE Task Force on Classification and Terminology. Epilepsia 2001; 42: 1212-1218.

7. Berg AT et al. Revides terminology and concepts for organization of seizures and epilepsies: Report of the ILAE Commission on Classification and Terminology, 2005-2009. Epilepsia 2010; 51: 676-685.

8. Lüders HO, Turnbull J, Kaffashi F. Are the dichotomies generalized versus focal epilepsies and idiopathic versus symptomatic epilepsies still valid in modern epileptology? Epilepsia 2009; 50: 1336-43
9. Wellmer J et al. Proposal for a magnetic resonance imaging protocol for the detection of epileptogenic lesions at early outpatient stages. Epilepsia 2013. 54: 1977-1987.

10. Von Oertzen J et al. Standard magnetic resonance imaging is inadequate for patients with refractory focal epilepsy. J Neurol Neurosurg Psychiatry 2002; 73: 643-647.

11. Steinhoff BJ et al. The impact of guidelines on the quality of MRI diagnostics in adult patients referred to a tertiary Epilepsy Centre. J Neurol 2013; 260: 2174-2175.

12. Steinhoff BJ. Antikonvulsive Pharmakotherapie Jugendlicher und Erwachsener. State of the art. Z Epileptol 2013; 26: 142-153.

13. Schmidt D. Pharmacotherapy of epilepsy - current problems and controversies. Fortschr Neurol Psychiat 1983; 51: 363-86

14. Schmidt D. Drug treatment of epilepsy: Options and limitations. Epilepsy Behav 2009; 15: 56-65

15. Baumgartner $\mathrm{C}$ et al. Erster epileptischer Anfall und Epilepsien im Erwachsenenalter. In: Diener HC, Weimar C, Berlit P et al. (Hrsg) Leitlinien für Diagnostik und Therapie in der Neurologie. Stuttgart: Thieme 2012, 28-47.

16. Steinhoff BJ, Kurth C, Dennig D. Levetiracetam bei generalisierten Epilepsien des Erwachsenenalters. Praktische Erfahrungen mit dem Einsatz außerhalb der Zulassung. Z Epileptol 2012; 25: 118-24.

17. Tomson $T$ et al. Valproate in the treatment of epilepsy in girls and women of childbearing potential. Epilepsia 2015; 56: 1006-1019.

18. Baker $\mathrm{G}$ et al. IQ at 6 years after in utero exposure to antiepileptic drugs: a controlled cohort study. Neurology 2015; 84: 382-390.

19. Steinhoff BJ. Der Epilepsiepatient in der Praxis. Basel: ComMed Verlagsagentur 2005.

20. Callaghan BC, Anand K, Hesdorffer D, Hauser WA, French JA. Likelihood of seizure remission in an adult population with refractory epilepsy. Ann Neurol 2007; 62(4): 382-9.

21. Luciano AL, Shorvon SD. Results of treatment changes in patients with apparently drug-resistant chronic epilepsy. Ann Neurol 2007; 62(4): 375-81.

22. Steinhoff BJ, Staack AM, Wisniewski I. Seizure control with antiepileptic drug therapy in 517 consecutive adult outpatients at the Kork Epilepsy Centre. Epileptic Disord 2012; 14: 379-387.

23. Rosenow $\mathrm{F}$ et al. The LaLiMo trial: lamotrigine compared with levetircatem in the initial 26 weeks of monotherapy for focal and generalised epilepsy - an open-label, prospective, randomised controlled multicenter Study. J Neurol Neurosurg Psychiatry 2012; 83: 1093-8.

24. Trinka E et al. KOMET: an unblinded, randomised, two parallel-group, stratified trial comparing the effectiveness of levetiracetam with controlledrelease carbamazepine and extended-release sodium valproate as monotherapy in patients with newly diagnosed epilepsy. J Neurol Neurosurg Psychiatry 2013; 84: 1138-1147.

25. Privitera MD et al. Topiramate, carbamazepine and valproate monotherapy: double-blind comparison in newly diagnosed epilepsy. Acta Neurol Scand 2003; 107: 165-175.
26. Steinhoff BJ et al. The LAM-SAFE Study: Lamotrigine versus carbamazepine or valproic acid in newly diagnosed focal and generalized epilepsies in adolescents and adults. Seizure 2005; 14: 597-605.

27. Rowan AJ et al. New onset geriatric epilepsy: a randomized study of gabapentin, lamotrigine and carbamazepine. Neurology 2005; 64: 1868-1873.

28. Ficker DM et al. Improved tolerability and efficacy in epilepsy patients with extended-release carbamazepine. Neurology 2005; 65: 593-5.

29. Saetre E et al. An international multicenter randomized double-blind controlled trial of lamotrigine and sustained-release carbamazepine in the treatment of newly diagnosed epilepsy in the elderly. Epilepsia 2007; 48: 1292-1302.

30. Werhahn $\mathrm{K}$ et al. A randomized, double-blind comparison of antiepileptic drug treatment in the elderly with new-onset focal epilepsy. Epilepsia 2015; 56: 450-459.

31. Doeser A et al. Targeting pharmacoresistant epilepsy and epileptogenesis with a dual-purpose antiepileptic drug. Brain 2015; 138: 371-387.

32. Klitgaard $\mathrm{H}$ et al. Brivaracetam: Rationale for discovery and preclinical profile of a selective SV2A ligand for epilepsy treatment. Epilepsia 2016; 57: 538-548.

33. Doty P et al. Development of lacosamide for the treatment of partial-onset seizures. Ann N Y Acad Sci 2013; 1291: 56-68.

34. Faulkner MA, Burke RA. Safety profile of two novel antiepileptic agents approved for the treatment of refractory partial seizures: ezogabine (retigabine) and perampanel. Expert Opin Drug Saf 2013; 12: 847-855.

35. Nass RD et al. Adjunctive retigabine in refractory focal epilepsy: Postmarketing experience at four tertiary epilepsy care centers in Germany. Epilepsy Behav 2016; 56: 54-58.

36. Correia FD et al. Two-year follow-up with eslicarbazepine acetate: a consecutive, retrospective, observational study. Epilepsy Res 2014; 108: 1399-1405.

37. Villanueva $\mathrm{V}$ et al. Long-term safety and efficacy of eslicarbazepine acetate in patients with focal seizures: results of the 1-year ESLIBASE retrospective study. Epilepsy Res 2014; 108: 1243-1252.

38. Runge $\mathrm{U}$ et al. A noninterventional study evaluating the effectiveness and safety of lacosamide added to monotherapy in patients with epilepsy with partial-onset seizures in daily clinical practice: The VITOBA study. Epilepsia 2015; 56: 1921-1930.

39. Trinka E, Steinhoff BJ, Nikanorova M, Brodie MJ. Perampanel for focal epilepsy: insights from early clinical experience. Acta Neurol Scand 2016; 133: 160-172.

40. Bauer $\mathrm{S}$ et al. Transcutaneous vagus nerve stimulation (tVNS) for treatment of drug-resistant epilepsy: A randomized, double-blind clinical trial (cMPsE02). Brain Stimul 2016; EPUB ahead of print.

41. Soss J et al. A prospective long-term study of external trigeminal stimulation for drug-resistant epilepsy. Epilepsy Behav 2015; 42: 44-47. 